\title{
Chagas disease reactivation in a heart transplant patient infected by domestic Trypanosoma cruzi discrete typing unit I ( $\mathrm{TCl}_{\mathrm{DOM}}$ )
}

\author{
Jaime A. Costales ${ }^{1 *}$, Camille N. Kotton², Andrea C. Zurita-Leal ${ }^{1,3}$, Josselyn Garcia-Perez ${ }^{1,4}$, Martin S. Llewellyn ${ }^{5,6}$, \\ Louisa A. Messenger ${ }^{5}$, Tapan Bhattacharyya ${ }^{5}$ and Barbara A. Burleigh ${ }^{7}$
}

\begin{abstract}
Background: Trypanosoma cruzi, causative agent of Chagas disease, displays high intraspecific genetic diversity: six genetic lineages or discrete typing units (DTUs) are currently recognized, termed Tcl through TcVl. Each DTU presents a particular distribution pattern across the Americas, and is loosely associated with different transmission cycles and hosts. Several DTUs are known to circulate in Central America. It has been previously suggested that Tcl infection is benign and does not lead to chronic chagasic cardiomyopathy (CCC).

Findings: In this study, we genotyped T. cruzi parasites circulating in the blood and from explanted cardiac tissue of an El Salvadorian patient who developed reactivation Chagas disease while on immunosuppressive medications after undergoing heart transplant in the U.S. as treatment for end-stage CCC. Parasite typing was performed through molecular methods (restriction fragment length polymorphism of polymerase reaction chain amplified products, microsatellite typing, maxicircle sequence typing and low-stringency single primer PCR, [LSSP-PCR]) as well as lineage-specific serology. We show that the parasites infecting the patient belong to the Tcl DTU exclusively. Our data indicate that the parasites isolated from the patient belong to a genotype frequently associated with human infection throughout the Americas ( $\mathrm{Cl}_{\mathrm{DO}}$ ).

Conclusions: Our results constitute compelling evidence in support of TCl DTU's ability to cause end-stage CCC and help dispel any residual bias that infection with this lineage is benign, pointing to the need for increased surveillance for dissemination of this genotype in endemic regions, the USA and globally.
\end{abstract}

Keywords: Trypanosoma cruzi, Lineage, Reactivation, Chagasic cardiomyopathy, Chagas disease

\section{Findings}

Chagas disease, caused by Trypanosoma cruzi, is the most important parasitic disease in Latin America, [1] and constitutes an emerging global public health problem, since thousands of T. cruzi- infected Latin Americans migrated during the last few decades and now live in North America, Europe, Australia, Japan and other regions [2]. A spectrum of clinical manifestations may result from human infection with $T$. cruzi, ranging from a total absence of symptoms to extremely debilitating and often deadly cardiac or digestive syndromes [3], and T. cruzi's genetic diversity is suspected

\footnotetext{
* Correspondence: jacostalesc@puce.edu.ec

'Centro de Investigación en Enfermedades Infecciosas, Escuela de Biología, Pontificia Universidad Católica del Ecuador, Avenida 12 de Octubre y Roca, Quito, Ecuador

Full list of author information is available at the end of the article
}

to influence the clinical outcome. Six major genetic lineages or discrete typing units (DTUs) are currently recognized (named TcI through $\mathrm{TcVI}$ ), each displaying different biological characteristics [4]. There are as yet no proven associations between $T$. cruzi genetic lineages and the clinical presentations of the disease; DTUs TcII, V and VI are frequently reported to be present in the Southern Cone of South America where serious chronic manifestations include megaesophagus and megacolon, whereas TcI is reported to predominate in endemic countries north of the Amazon [4]. The pathogenicity of this lineage is evident in acute cases, where patent clinical manifestations, including death, have been reported in TcI oral outbreaks [5]. However, in terms of chronic symptoms, TcI has been suggested to be benign [6], with observed chronic 


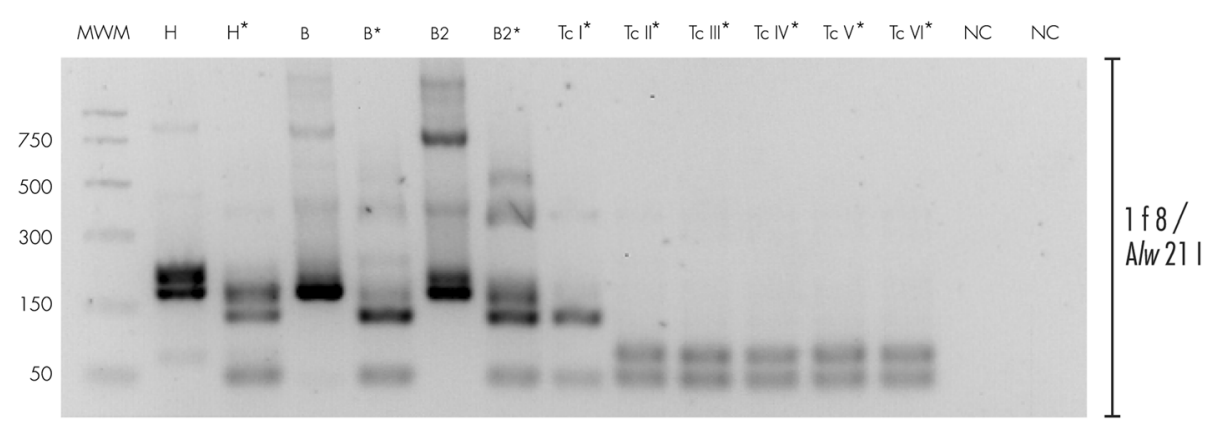

Fig. 1 Molecular typing from clinical samples. DNA was extracted from blood samples and parafinized heart explants. Typing was performed using a nested PCR-RFLP strategy for amplification of the $1 \mathrm{f} 8$ flagellar protein and digestion with Alw 211 (Van der Auwera, et al., unpublished data). H= DNA extracted from parafinized heart explant tissue, $\mathrm{B}$ and $\mathrm{B} 2=$ blood samples taken 10 days apart during reactivation of disease, Tcl-TcVl correspond to DTU controls, NC lanes correspond to negative controls for the PCR and nested-PCR. Lanes corresponding to Alw 211 restriction digest products are labeled with an asterisk ${ }^{*}$ ). Only restriction products are shown for controls

chagasic cardiomyopathy (CCC) in TcI-infected patients attributed to co-infection with other T. cruzi DTUs.

Despite being based on limited sampling, this view has been reiterated in additional publications [7-9]. Furthermore, it has also been suggested that Chagas disease may be less severe among patients in the Amazon basin, where TcI infection is likely [10]. TcI exhibits high intra-lineage diversity, with members of a widely dispersed subgroup (termed $\mathrm{TCI}_{\mathrm{DOM}}$ ) associated with many human infections [11], although no direct link with clinical manifestations has been established.

Here, we report a case of chronic chagasic cardiomyopathy and reactivation disease accompanied by extensive molecular characterization of the T. cruzi parasites involved. A 43 year old chagasic patient from El Salvador was admitted to Massachusetts General Hospital to undergo orthotropic heart transplantation.
Immunofluorescence assay to detect anti-T. cruzi IgG previously performed at the Centers for Disease Control \& Prevention (CDC, Atlanta, USA) was positive at $>1: 256$ (diagnostic threshold $>1: 32$ ). His cardiac symptoms corresponded to New York Heart Association class IV and he had a biventricular pacemaker/defibrillator for complete heart block. Fifteen months previously, he underwent mitral valve replacement, tricuspid valve reconstruction and partial left ventriculectomy, at which time a left ventricle excision and atrial appendage biopsy showed active and healed myocarditis, consistent with late phase Chagas disease. He had no other known etiologies for his heart failure. Orthotropic heart transplantation was successful. Pathologic examination of the explanted heart revealed findings consistent with end-stage CCC, including dilatation with near complete atrophy of the left ventricular wall, endocardial fibrosis, diffuse myocardial

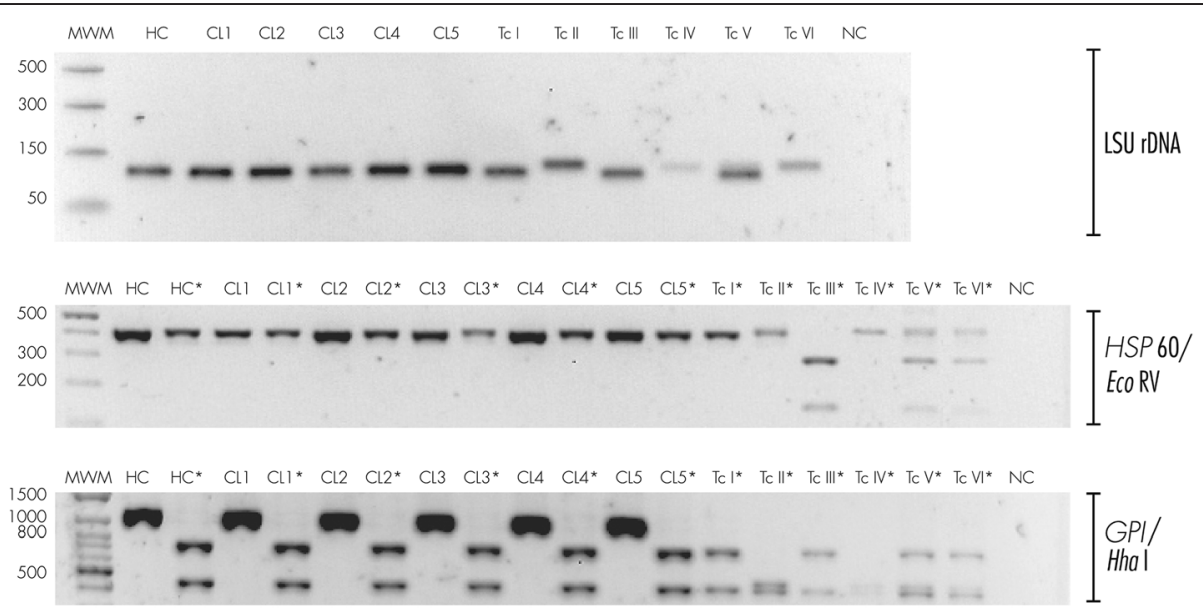

Fig. 2 Molecular typing for cultured parasites and parasite clones. DNA was extracted from epimastigote hemocultures (HC) and five derived clones (CL1-CL5). TCl-TCVI correspond to DTU controls, NC corresponds PCR negative control. Lanes containing restriction products are labeled with an asterisk $\left(^{*}\right)$. Only restriction products are shown for controls. DNA was analyzed by the PCR-RFLP scheme proposed by Lewis, et al., 2009 [12]: as indicated by the brackets on the right side, fragments from the LSUrDNA, HSP60 and GPI genes were amplified by PCR. GPI and HSP60 products were digested with Hhal and EcoRV restriction enzimes, respectively 


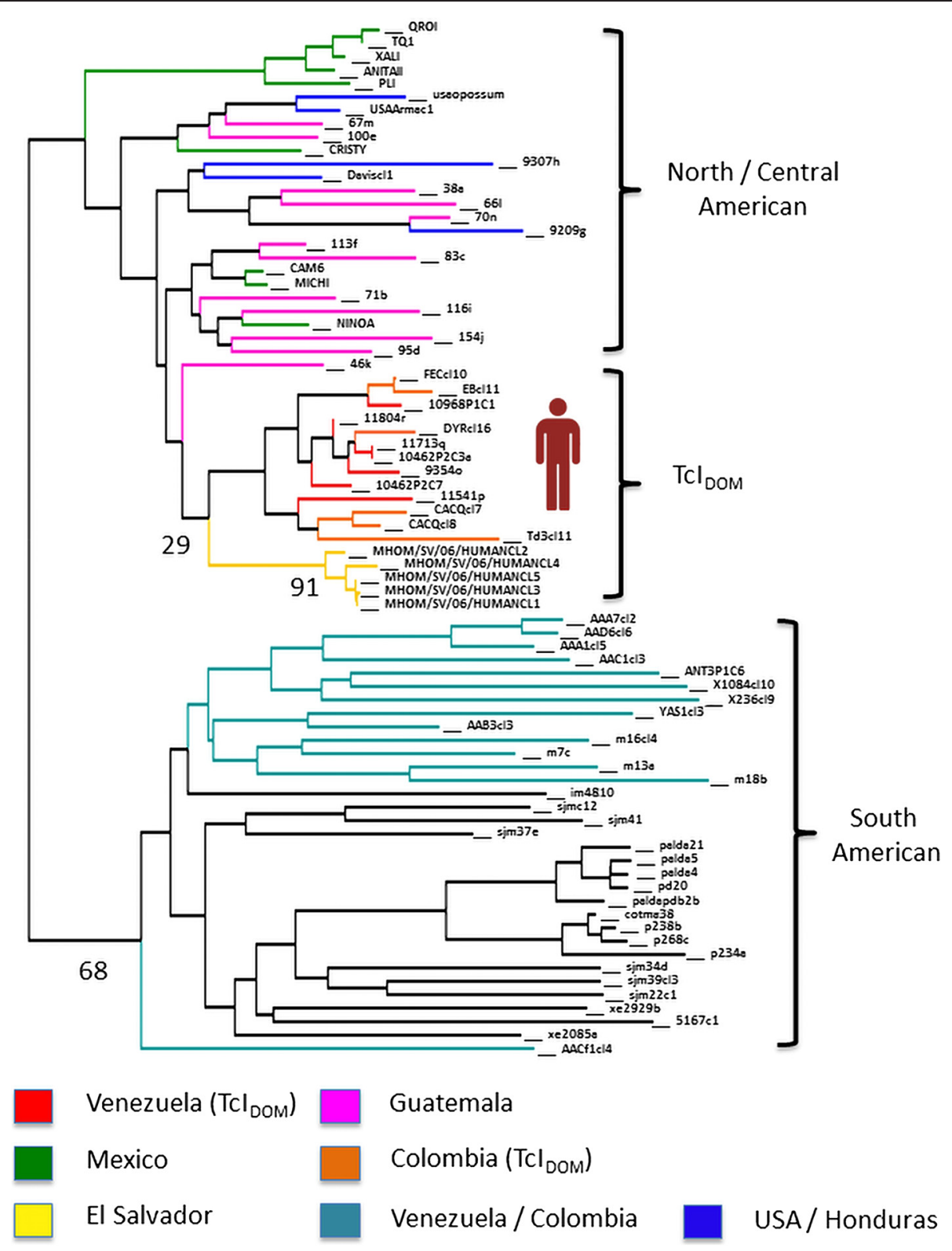

Fig. 3 Neighbour joining dendrogram based on pairwise inverse allele sharing. Relationship between parasite clones isolated from the patient and others from North, Central and South America are shown. Branch colours indicate strain origin and values at important nodes indicate percentage of bootstrap support over 1000 trees. Further details of strains and analytical strategy can be found in [11]

fibrosis, and mononuclear infiltrates with some eosinophils and neutrophils. The infiltrate (lymphocytic myocarditis) was composed of lymphocytes (many CD3 T cells, more CD8 than CD4, few CD20 B cells) plus many CD68 macrophages. Amastigotes were not identified on multiple sections examined; however, $T$. cruzi kinetoplast DNA was detected by PCR in frozen tissue submitted to the Parasitic Diseases and Diagnostics Branch of the CDC. Weekly microscopic examination of his blood was performed after transplant, screening for early detection of reactivation disease; this was positive with rare trypomastigotes detected at week six after transplant. He was given nifurtimox $8-10 \mathrm{mg} / \mathrm{kg}$ (as $120 \mathrm{mg}$ four times a day) orally for 10 weeks, and developed severe peripheral neuropathy with anorexia. $\mathrm{He}$ was switched to benznidazole, $150 \mathrm{mg}$ twice a day for 30 days, which he tolerated well, and his peripheral neuropathy and anorexia resolved. He was monitored monthly for parasitemia for approximately one year after the end of therapy, with no evidence of further infection.

Genotyping directly from patient's blood samples and parafinized heart explants using a nested PCR-RFLP for the 


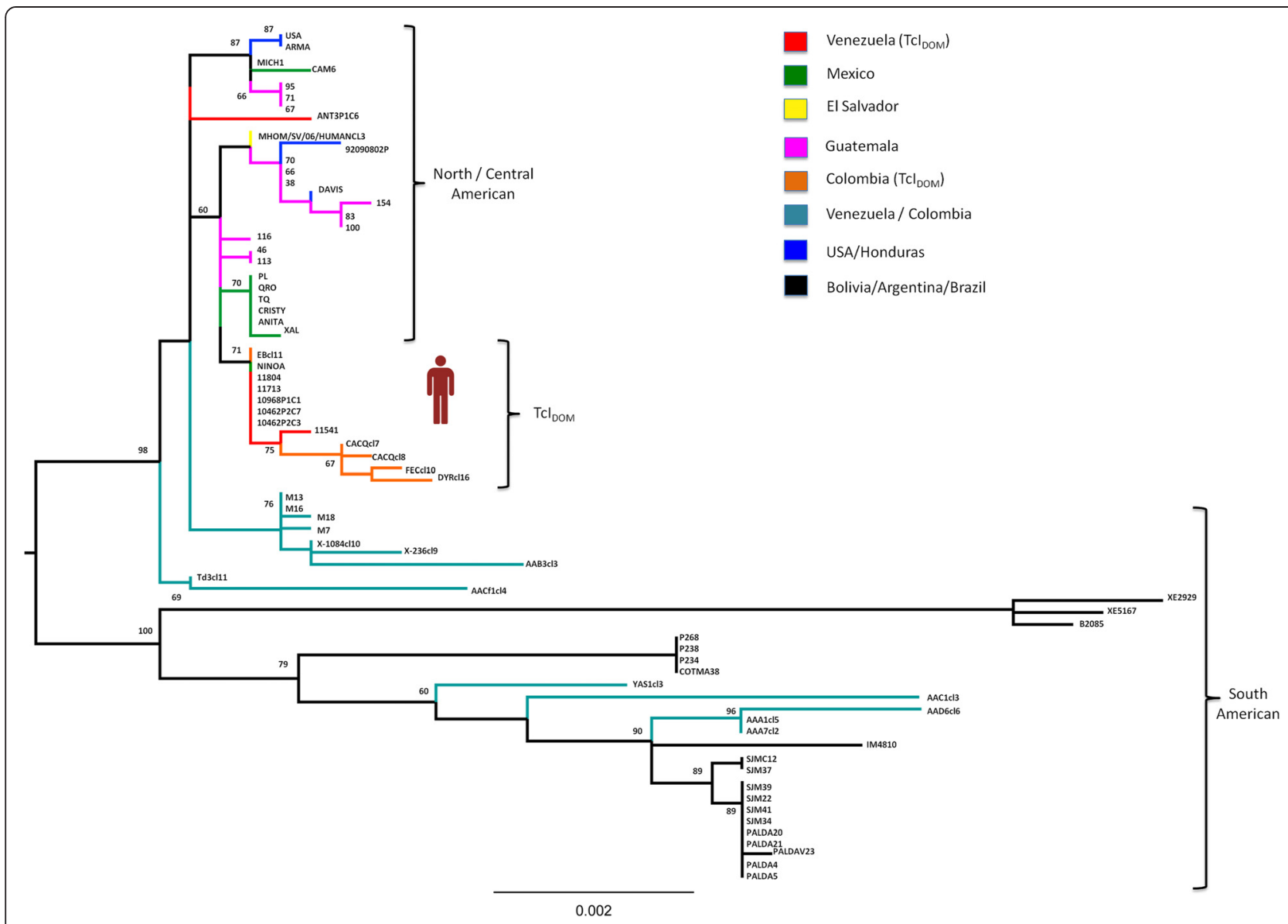

Fig. 4 Maxicircle sequence-based typing of strain isolated from patient. Maxicircle sequences for one biological clone were concatenated (accession \# KP136828) according to [14], aligned against 70 Tcl strains encompassing Tcl genetic diversity from across North, Central and South America [11] and used to assemble a Maximum-Likelihood topology in PhyML. The best-fit model of nucleotide substitution was selected from 88 models and its significance evaluated according to the Akaike Information Criterion (AIC) in jMODELTEST 1.0. The best fit model selected for this dataset was GTR + G. Bootstrap support for clade topologies was estimated following the generation of 100 pseudo-replicate datasets

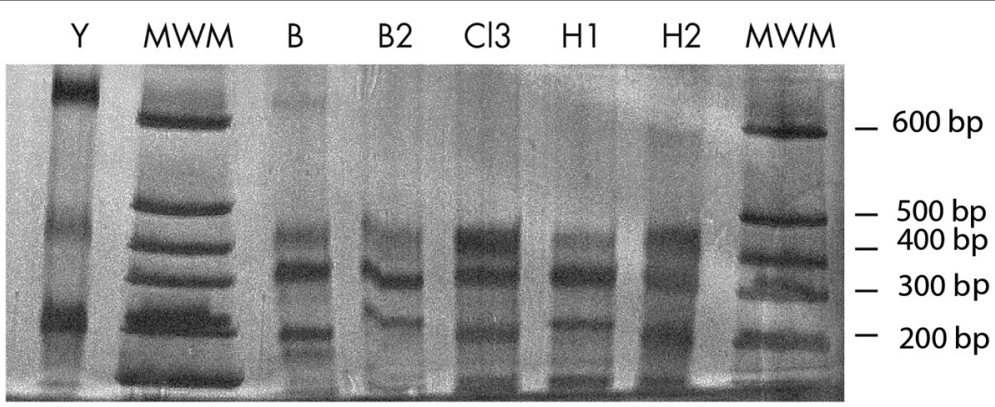

Fig. 5 LSSP-PCR. DNA from parafinized heart explants and blood samples was amplified using the S35/S36 primer pair. The 330 bp product was gel-purified and analyzed by LSSP-PCR. Products were run in a $10 \%$ polyacrilamide gel and silver-stained. $\mathrm{H} 1$ and $\mathrm{H} 2$ correspond to DNA extracted from two different sections of parafinized heart explant tissue B and B2 correspond to blood samples taken 10 days apart, starting six weeks after transplant. $\mathrm{Cl} 3$ corresponds to DNA from one of the clones derived from the original T. cruziisolate. Y indicates "Y strain" parasites (Tcll DTU) used for comparison. $\mathrm{MWM}=$ molecular weight markers 
1f8 flagellar protein and digestion with Alw21I restriction enzyme (Van der Auwera, unpublished) assigned parasites to DTU TcI (Fig. 1). Hemoculture six and eight weeks after transplant yielded epimastigotes, which were cloned in solid medium. Cultured parasites and clones were determined to belong to DTU TcI by polymerase chain reaction and restriction fragment length polymorphism (PCR-RFLP) as in [12] (Fig. 2). Intra-TcI genotyping was performed with nuclear microsatellites [13] and maxicircle gene fragments [14]. Intriguingly, microsatellite data indicate a close relationship with $\mathrm{TCI}_{\mathrm{DOM}}$, a distinct genotype within DTU TcI which is common among human cases in Latin America (Fig. 3), while the maxicircle sequence analysis indicates an origin among wild/non-human isolates for North and Central America (Fig. 4). Additionally, LSSP-PCR [15] showed matching patterns among DNA extracted from patient blood samples, heart explants and cultured parasite clones, indicating that they represent the same parasite population. Meanwhile, Y-strain, from the TcII lineage, employed as a control, yielded a completely different amplification pattern (Fig. 5).

Finally, although patient's serum reacted against $T$. cruzi antigen in three different commercial serological tests (Chagatek-Biomerieux, Chagas III-Abbott BiosChile and Chagatest Recombinante 3.0-Wiener Lab), it did not recognize synthetic peptides derived from the TSSA antigen specific for DTUs TcII, III, IV or V/VI described in [16]. Therefore, we conclude that the observed CCC was caused by TcI, unequivocally confirming that $\mathrm{TcI}$ is an agent of life threatening heart disease.

TCI constitutes the most abundant and widespread $T$. cruzi DTU [4] and is the predominant DTU in the Amazon region and countries North of it [1]. For our patient, only TcI DTU parasites were detectable in the clinical samples, hemocultures, and biologically cloned parasites from hemoculture. Furthermore, antibodies against TcII, III, IV or V/ VI - specific peptide epitopes were not detectable in serum, suggesting the absence of co-infection or previous infection with those lineages; there is currently no adequately sensitive equivalent TcI-specific serological assay [16].

TcI has considerable intra-DTU diversity [13]; specific genotypes within TcI are associated with human infection [11]. Based on nuclear microsatellite information, the patient was infected by $\mathrm{TCI}_{\mathrm{DOM}}$, a genotype associated with many human infections in regions north of the Amazon. $\mathrm{TCI}_{\mathrm{DOM}}$ has not previously been reported from cardiac tissues in CCC cases, as it has been merely detected in peripheral blood or hemoculture, where co-infection with parasites from other genetic lineages causing CCC cannot be ruled out. Remarkably, mitochondrial genotyping suggests a closer relationship with isolates from North and Central America, consistent with local, possibly sylvatic, origin of the infecting strain. Given the proclivity for mitochondrial introgression into $\operatorname{TcI}_{\mathrm{DOM}}[11,14,17,18]$ we suggest our observation represents yet another such hybrid among many, in this case between $\mathrm{TCI}_{\mathrm{DOM}}$ and a local strain, highlighting the need for control strategies aimed at domiciliary and extrinsic parasite populations as sources of human infection.

The case portrayed herein highlights many of the challenges that may arise when encountering a case of CCC (end-stage heart failure, need for heart transplant, Chagas disease reactivation under immune suppression, adverse reaction to parasiticidal treatment) which were very competently handled by a capable medical team in a non-endemic nation. Awareness of Chagas disease as an emerging global problem is essential for successfully meeting such challenges, which are predicted to be faced with increased frequency in non-endemic countries.

Furthermore, the results of our molecular characterization constitute compelling evidence in support of the role of TcI as causative agent of end-stage CCC and help dispel any residual bias that infection with this lineage is benign. Considering the broad distribution of TcI (the only $T$. cruzi DTU ranging from the Southern United States to Argentina and Chile) and the frequency with which TcI strains are associated with human infection [4], there is need for greater surveillance in $\mathrm{TcI}$ endemic regions like Central America. Around 22 million people from Chagas endemic countries live in the US, and most of these immigrants come from Mexico (74 \%) and El Salvador (6.4 \%) [2], where TcI is known to predominate [4]. Thus, a significant proportion of the estimated 300,000 T. cruzi infections among immigrants in the U.S.A. are predicted to involve the DTU TcI, adding to the growing economic burden of medical care and interventions associated with Chagas disease in the U.S.A., including transplantation for endstage heart disease.

\section{Ethical approval}

The study was approved by MGH's Internal Review Board and written informed consent was obtained from the patient.

\section{Abbreviations}

CCC: Chronic chagasic cardiomyopathy; DTU: Discrete typing unit; Tcl: Trypanosoma cruzi lineage I; Tcl Trypanosoma cruzi lineage I frequently associated with human infection; LSSP-PCR: Low stringency single primer polymerase chain reaction.

\section{Competing interests}

The authors declare that they have no competing interests.

Authors' contributions

JAC conceived and coordinated the study, isolated parasites and performed molecular characterization, drafted the manuscript; CNK managed the clinical case; ACZ, JEG, MSL and LAM performed molecular characterization; TB performed lineage specific serology; BAB conceived the study and helped draft the manuscript. All authors read and approved the final manuscript. 


\section{Acknowledgments}

We thank Fernanda Latorre, Alejandra Zurita for technical assistance, Gert Van der Auwera for valuable discussion of the data, Mario Grijalva and Michael Miles for critical reading of the manuscript. Work was funded by PUCE.

\section{Author details}

${ }^{1}$ Centro de Investigación en Enfermedades Infecciosas, Escuela de Biología, Pontificia Universidad Católica del Ecuador, Avenida 12 de Octubre y Roca, Quito, Ecuador. ${ }^{2}$ Division of Infectious Diseases, Massachusetts General Hospital, Boston, USA. ${ }^{3}$ Present address: Wellcome Trust Centre for Molecular Parasitology, Institute of Infection and Inflammation, College of Medical, Veterinary and Life Science, University of Glasgow, Glasgow, UK. ${ }^{4}$ Present address: VIB Autoimmune Genetics Laboratory, Department of Microbiology and Immunology, KU Leuven, Leuven, Belgium. ${ }^{5}$ Department of Pathogen Molecular Biology, London School of Hygiene and Tropical Medicine, London, UK. ${ }^{6}$ Present address: Molecular Ecology and Fisheries Genetics Laboratory, School of Biological Sciences, University of Wales, Bangor, Deiniol Road, Bangor, Gwynedd LL57 2UW, UK. ${ }^{7}$ Department of Immunology and Infectious Diseases, Harvard School of Public Health, Boston, USA.

\section{Received: 10 March 2015 Accepted: 10 August 2015}

\section{Published online: 25 August 2015}

\section{References}

1. Miles MA, Llewellyn MS, Lewis MD, Yeo M, Baleela R, Fitzpatrick S, et al. The molecular epidemiology and phylogeography of Trypanosoma cruzi and parallel research on Leishmania: looking back and to the future. Parasitology. 2009;136(12):1509-28.

2. Gascon J, Bern C, Pinazo MJ. Chagas disease in Spain, the United States and other non-endemic countries. Acta Trop. 2010;115(1-2):22-7.

3. Rassi Jr A, Rassi A, Marin-Neto JA. Chagas disease. Lancet 2010;375(9723):1388-402.

4. Zingales B, Miles MA, Campbell DA, Tibayrenc M, Macedo AM, Teixeira MM et al. The revised Trypanosoma cruzi subspecific nomenclature: rationale, epidemiological relevance and research applications. Infect Genet Evol. 2012;12(2):240-53.

5. Diaz-Bello Z, Thomas MC, Lopez MC, Zavala-Jaspe R, Noya O, Den BA, et al. Trypanosoma cruzi genotyping supports a common source of infection in a school-related oral outbreak of acute Chagas disease in Venezuela. Epidemiol Infect. 2014;142(1):156-62.

6. Di Noia JM, Buscaglia CA, De Marchi CR, Almeida IC, Frasch AC. A Trypanosoma cruzi small surface molecule provides the first immunological evidence that Chagas' disease is due to a single parasite lineage. J Exp Med. 2002;195(4):401-13.

7. Barrett MP, Burchmore RJ, Stich A, Lazzari JO, Frasch AC, Cazzulo JJ, et al The trypanosomiases. Lancet. 2003;362(9394):1469-80.

8. Morel CM, Lazdins J. Chagas disease. Nat Rev Microbiol. 2003;1(1):14-5.

9. Buscaglia CA, Di Noia JM. Trypanosoma cruzi clonal diversity and the epidemiology of Chagas' disease. Microbes Infect. 2003;5(5):419-27.

10. Coura JR, Borges-Pereira J. Chagas disease. What is known and what should be improved: a systemic review. Rev Soc Bras Med Trop. 2012;45(3):286-96.

11. Zumaya-Estrada FA, Messenger LA, Lopez-Ordonez T, Lewis MD, Flores-Lopez CA, Martinez-lbarra AJ, et al. North American import? Charting the origins of an enigmatic Trypanosoma cruzi domestic genotype. Parasit Vectors. 2012;5:226.

12. Lewis MD, Ma J, Yeo M, Carrasco HJ, Llewellyn MS, Miles MA. Genotyping of Trypanosoma cruzi: systematic selection of assays allowing rapid and accurate discrimination of all known lineages. Am J Trop Med Hyg. 2009;81(6):1041-9.

13. Llewellyn MS, Miles MA, Carrasco HJ, Lewis MD, Yeo M, Vargas J, et al. Genome-scale multilocus microsatellite typing of Trypanosoma cruz discrete typing unit I reveals phylogeographic structure and specific genotypes linked to human infection. PLoS Pathog. 2009;5(5), e1000410.

14. Messenger LA, Llewellyn MS, Bhattacharyya T, Franzen O, Lewis MD, Ramirez $J D$, et al. Multiple mitochondrial introgression events and heteroplasmy in trypanosoma cruzi revealed by maxicircle MLST and next generation sequencing. PLoS Negl Trop Dis. 2012;6(4), e1584.

15. Mejia AM, Triana O. [Genetic variability of Trypanosoma cruzi in blood and organs of infected mice determined by LSSP-PCR]. Biomedica. 2005;25(1):76-86.

16. Bhattacharyya T, Falconar AK, Luquetti AO, Costales JA, Grijalva MJ, Lewis MD, et al. Development of peptide-based lineage-specific serology for chronic Chagas disease: geographical and clinical distribution of epitope recognition. PLoS Negl Trop Dis. 2014;8(5), e2892.

17. Ramirez JD, Guhl F, Messenger LA, Lewis MD, Montilla M, Cucunuba Z, et al. Contemporary cryptic sexuality in Trypanosoma cruzi. Mol Ecol. 2012;21(17):4216-26.

18. Lima VS, Jansen AM, Messenger LA, Miles MA, Llewellyn MS. Wild Trypanosoma cruzi I genetic diversity in Brazil suggests admixture and disturbance in parasite populations from the Atlantic Forest region. Parasit Vectors. 2014;7:263.

\section{Submit your next manuscript to BioMed Central and take full advantage of:}

- Convenient online submission

- Thorough peer review

- No space constraints or color figure charges

- Immediate publication on acceptance

- Inclusion in PubMed, CAS, Scopus and Google Scholar

- Research which is freely available for redistribution

Submit your manuscript at www.biomedcentral.com/submit 